\begin{tabular}{|c|c|}
\hline Title & Measurements of dental cast profile and three dimensional tooth movement during orthodontic treatment \\
\hline Author(s) & Y amamoto, K.; Hayashi, S.; Nishikawa, H.; Nakamura, S.; Mikami, T. \\
\hline Citation & $\begin{array}{l}\text { IEEE Transactions on Biomedical Engineering, 38(4), 360-365 } \\
\text { https://doi.org/10.1109/10.133232 }\end{array}$ \\
\hline Issue Date & 1991-04 \\
\hline Doc URL & http:/hdl .handle.net/2115/6036 \\
\hline Rights & $\begin{array}{l}\text { C1991 IEEE. Personal use of this material is permitted. However, permission to reprint/republish this material for } \\
\text { advertising or promotional purposes or for creating new collective works for resale or redistribution to servers or lists, } \\
\text { or to reuse any copyrighted component of this work in other works must be obtained from the IEEE. } \\
\text { IEEE,IEEE Transactions on Biomedical Engineering, 38(4), 1991, p360-365 }\end{array}$ \\
\hline Tyре & article \\
\hline File Information & ITBE38_4.pdf \\
\hline
\end{tabular}

Instructions for use 


\title{
Measurements of Dental Cast Profile and Three- Dimensional Tooth Movement During Orthodontic Treatment
}

\author{
Katsuyuki Yamamoto, Member, IEEE, Syunsuke Hayashi, Hirofumi Nishikawa, \\ Shinji Nakamura, and Tomohisa Mikami
}

\begin{abstract}
We have developed a system for measuring three-dimensional profiles of dental casts and three-dimensional tooth movement during orthodontic treatment. The profile measurement is based on the triangulation method which detects a laser sport on a cast using an image sensor. The system is computer-controlled and designed to achieve the depth and lateral resolutions of 0.05 and $0.1 \mathrm{~mm}$, respectively, within a depth range of $25 \mathrm{~mm}$. We have applied our system to measuring three-dimensional movement of teeth, including rotations, during orthodontic treatment. The movements have been obtained by means of three-dimensional registration of tooth profiles using a computer between casts serially taken at different stages of the treatment. Measurements over periods of 41 to 190 days on patients have revealed that this technique is capable of quantifying the difference in movements due to orthodontic therapeutics.
\end{abstract}

\section{INTRODUCTION}

A dental cast is widely used in dentistry for working on dental prostheses. Because of the problems involved in working within the mouth, tooth shape and position, which provide fundamental diagnostic information in dentistry, are also generally measured on a dental cast. This is called cast analysis and is most frequently carried out by using simple instruments such as a caliper and a ruler. These instruments, however, allow only one-dimensional measurement. In order to measure a cast in all three dimensions, $X, Y$, and $Z$, instrumentations of indirect and direct measurements have been attempted. As for the indirect measurement of shape, stereophotogrammetry has been applied to the morphological study of the face, the tooth, and the palate [1]-[3]. Although this technique has been used successfully to measure three-dimensional shapes, it requires a sophisticated procedure to digitize the shape.

Direct measurements employing either a contacting mechanical or noncontacting optical technique have been attempted. Van der Linden et al. [4] have described the optocom which can measure $X$ and $Y$ coordinates using a microscope and a $Z$ coordinate by mechanical contact with a dental cast. The reflex optical plotter using a noncontacting optical technique has been applied to the measurement of all three coordinates [5], [6]. In this apparatus the measurement is made by aligning a movable light source to coincide with a specific point on a cast image behind a semireflecting mirror. A traveling microscope recently developed by Bhatia and Harrison [7] is based on an optical

Manuscript received January 19, 1989; revised January 27, 1990.

K. Yamamoto, H. Nishikawa and T. Mikami are with Division of Biomedical Engineering, Faculty of Engineering, Hokkaido University, Sapporo 060, Japan.

S. Hayashi and S. Nakamura are with Department of Orthodontics, School of Dentistry, Hokkaido University, Sapporo 060, Japan

IEEE Log Number 9144244 technique which has notably increased the accuracy of the measurement. However, in all cases, since the apparatus in manually operated point-to-point, little data concerning surface profiles can be obtained.

A computer-controlled profilometer using an optical probe has been developed for the measurement of cleft palate casts by Berkowitz et al. [8]. Although this apparatus scans a cast automatically and digitizes its profiles, the optical technique used does not meet the demands for the measurement of occlusal surface of a tooth because it has a fine profile. Greater spatial resolution is required for the measurement. The authors have developed a prototype of an automatic, optical profilometer equipped with a laser and an image sensor and capable of a higher degree of precision [9], [10].

In orthodontics, information concerning the positional change of a tooth is as fundamental and important as the shape. When an orthodontic force is applied to a tooth over a long period of time, the tooth moves owing to resorption of the alveolar bone on the pressurized side and apposition of new bone on the opposite side. In daily practice, orthodontists analyze tooth movement during treatment in a one-dimensional way by measuring the distance between two teeth using a caliper, or in a twodimensional way by tracing teeth on X-ray films taken at different stages of the treatment [11]. Although the tooth moves in a three-dimensional way, no established technique to measure this movement, including rotational movement, has been reported. If the three-dimensional shape of dental casts at the different stages is digitized into a computer, three-dimensional tooth movement can be obtained by numerical calculation. In this paper, we describe a system designed for the three-dimensional measurement of dental cast profiles and tooth movement during orthodontic treatment.

\section{Three-Dimensional Measurement of Dental CASTS}

\section{Instrumentation and Measurement}

A triangulation method is a conventional technique for the measurement of a distance. This method has recently been applied to noncontacting three-dimensional measurement using an optical probe. A biomedical application of this technique to the surface profile measurement of the trunk has been reported in recent years [12]. Based on a similar technique, we have developed a prototype of our system for measuring dental cast profiles [9], [10]. The measurement system including a personal computer is now more compact than the prototype, as shown in Fig. 1. The depth is measured with an optical system composed 


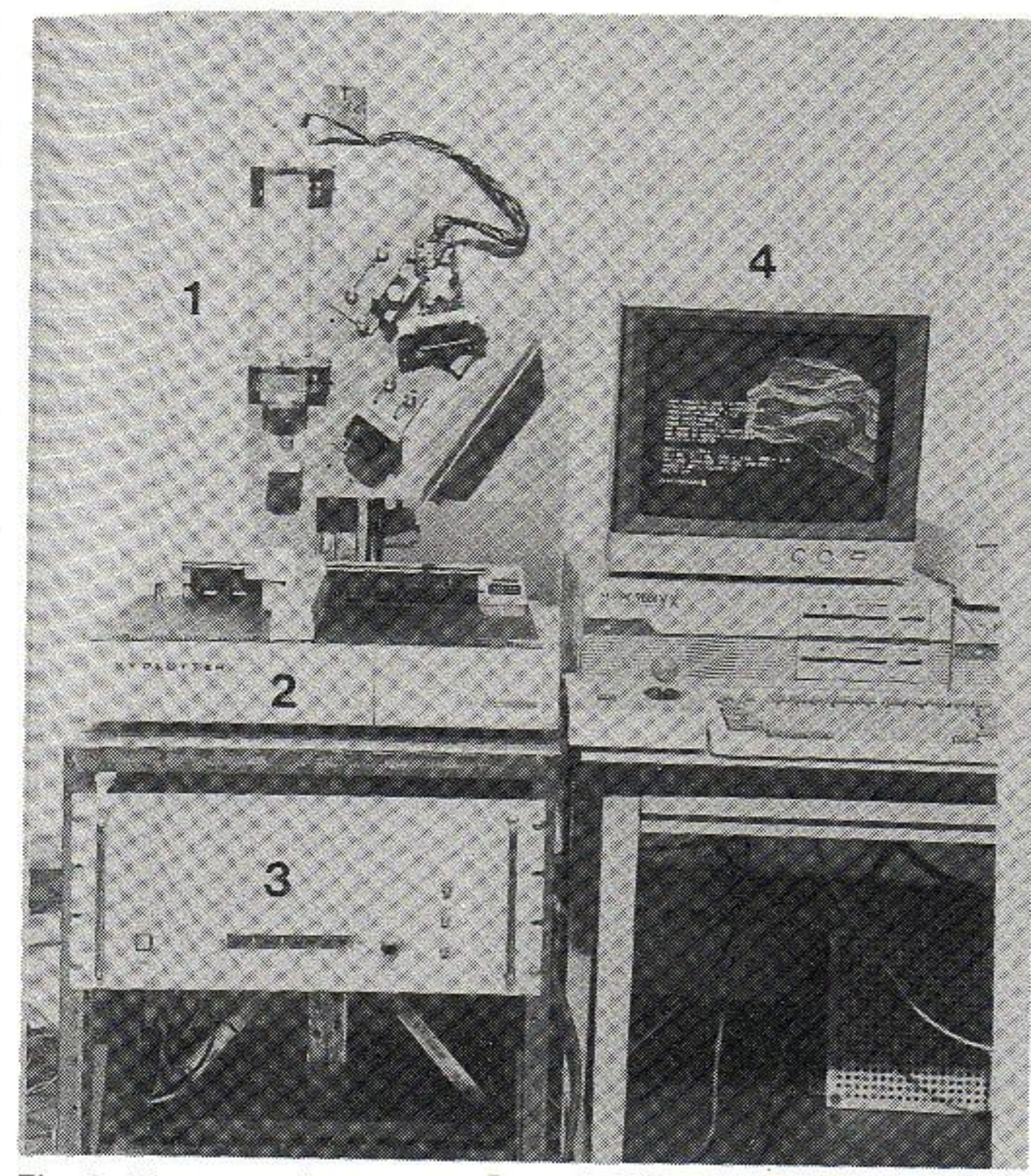

Fig. 1. The system developed for measuring dental cast profiles. The system is constructed with the optical equipment composed of a laser, an image sensor, and lenses (1), the $X-Y$ table (2), the specially designed interface with a personal computer (3), and the computer (4). The computer is also used for calculating three-dimensional tooth movement.

of a laser and an image sensor, as illustrated in Fig. 2. A small spot of light is formed on a dental cast with a $0.6 \mathrm{~mW} \mathrm{He}-\mathrm{Ne}$ laser and a lens $L_{1}(f=100 \mathrm{~mm})$. This lens is employed to sharpen the laser beam to a point in order to raise the power of lateral resolution, because the diameter $0.8 \mathrm{~mm}$ of the unfocused beam from the laser is too thick to measure the fine occlusal surface of a tooth. Scattered light from the spot is focused, with the other lens $L_{2}(f=75 \mathrm{~mm})$, onto an image sensor (Sharp LZ2020) which has a one-dimensional array of 2048 photoelements aligned in a pitch of $14 \mu \mathrm{m}$. From the position of the sport on the array the depth of the cast surface can be obtained. Scanning required for profile measurement is accomplished by moving the cast in a horizontal plane with an $X-Y$ table, for which a conventional $X-Y$ plotter (Graphtec FD5311) controlled by a personal computer (NEC PC-9801) was substituted for ease of instrumentation. The image sensor is placed on an optical axis $W$ making an angle of $\alpha\left(35^{\circ}\right)$ with the incident optical axis $Z$ of the laser beam. The sensor is also tilted by $\beta\left(45^{\circ}\right)$ with respect to the axis $W$ in such a way that the spot image is always focused on the array of photoelements.

The intensities of light stimulating 2048 photoelements are sequentially read from the sensor with a $1-\mathrm{MHz}$ clock and fed into a specially designed interface with the computer. The interface detects the rising and falling edges of the spot signal using a voltage comparator and obtains the position of the edges on the array, $P_{1}$ and $P_{2}$ shown in Fig. 2, by counting the clock pulses from the beginning of a read-out cycle to the edges. The two counted binary data are transferred to the computer via a digital input. The center of the two edges is determined and defined as the peak position, because it almost coincides with the actual position of the peak intensity. The peak position is

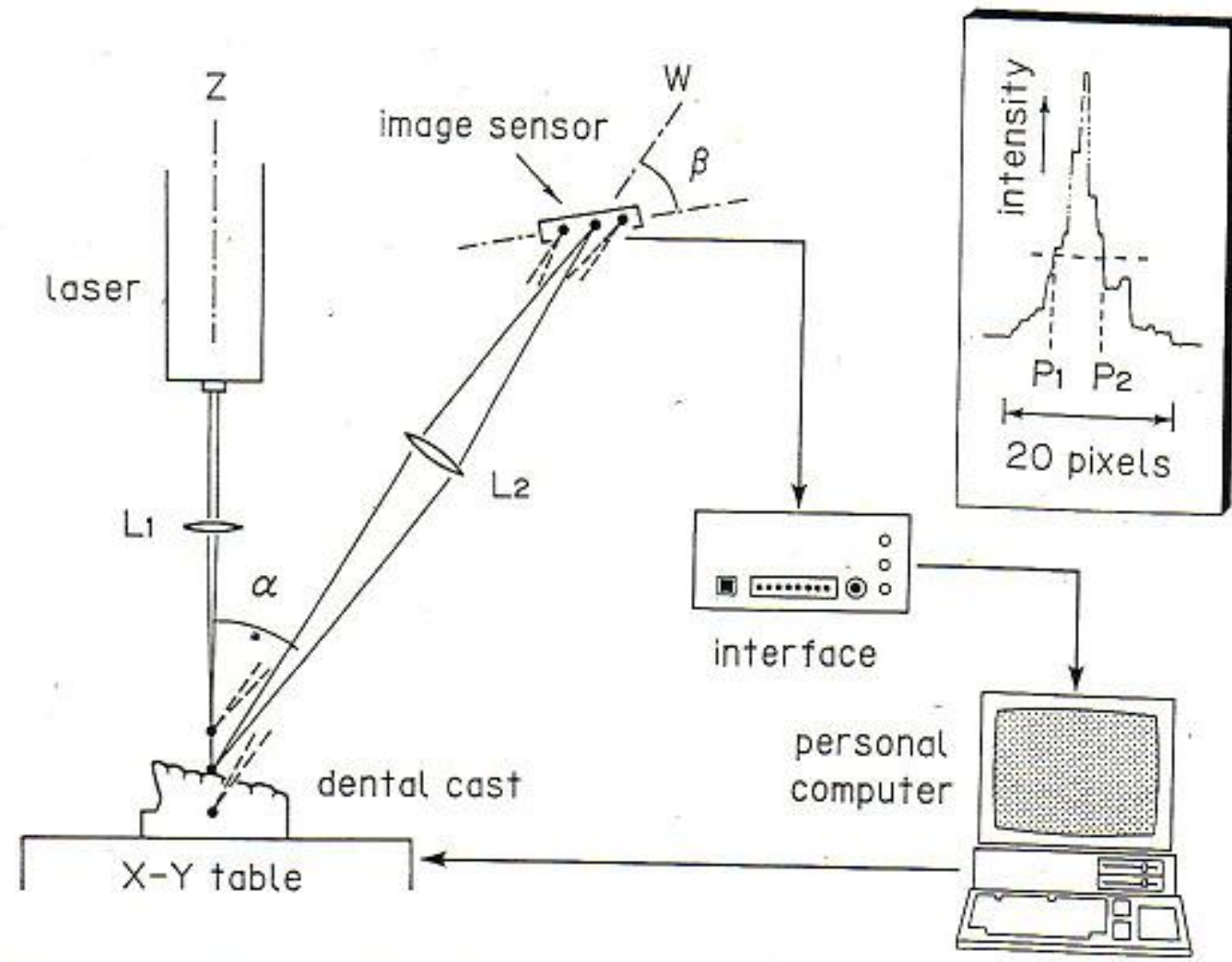

Fig. 2. Schematic diagram of the measuring system. A spot of light is formed with the laser and the lens $L_{1}$ on a dental cast placed on the $X-Y$ table. Cast profile is obtained from the position of the spot image formed by the lens $L_{2}$ onto the one-dimensional image sensor while scanning the cast. $\alpha$ and $\beta$ are $35^{\circ}$ and $45^{\circ}$, respectively. A spot signal from the image sensor distributes over about 20 pixels (photoelements) around the peak (right upper panel). The interface detects rising $(P 1)$ and falling $(P 2)$ portions and transfers their positions to the computer, in which the peak corresponding to depth is obtained.

then converted into the corresponding depth with a calibration curve obtained prior to the measurement, as described in the next section.

A plaster dental cast is fixed with pieces of double-sided adhesive tape on a platform supporting a plotter pen. The easiest way to scan a cast is to select one rectangular scanned area which covers all the teeth and the palate concerned. However, this method requires the complicated image processing for identifying the boundaries between tightly connected teeth. To eliminate this problem, the scanned area including the boundaries is predetermined on each tooth and the palate, prior to the measurement. An operator can easily adjust the scanned area with a joystick linked to the computer. After setting the scanned areas the apparatus automatically and successively digitizes teeth and palate profiles. Teeth and the palate were typically scanned at a pitch of 0.2 and $0.4 \mathrm{~mm}$, respectively. All the data are stored on a flexible disk and used for the calculation of tooth movement by the method described in the next chapter.

An example of the computer graphic display of measured profiles of a dental cast is shown in Fig. 3. The shape of the occlusal surface, which is necessary for the calculation of threedimensional tooth movement, is almost completely measured except for the lateral portion of the teeth. The lack of data in this portion is because the laser spot is not formed due to anatomical arrangement of the dental arch.

\section{Measurement Speed and Resolution}

The time required for measuring a point was $25 \mathrm{~ms}$ and was mostly consumed by mechanical scanning with the plotter. The number of sampled data per tooth amounts to nearly 2000 on the average, and it takes about $50 \mathrm{~s}$ per tooth.

We calibrated the apparatus and examined depth resolution in the following manner. Details have been described in our previous study [10]. The relationship between the peak position of the sensor and the depth of an object surface was measured by moving vertically, at a interval of $0.5 \mathrm{~mm}$ in the region of 


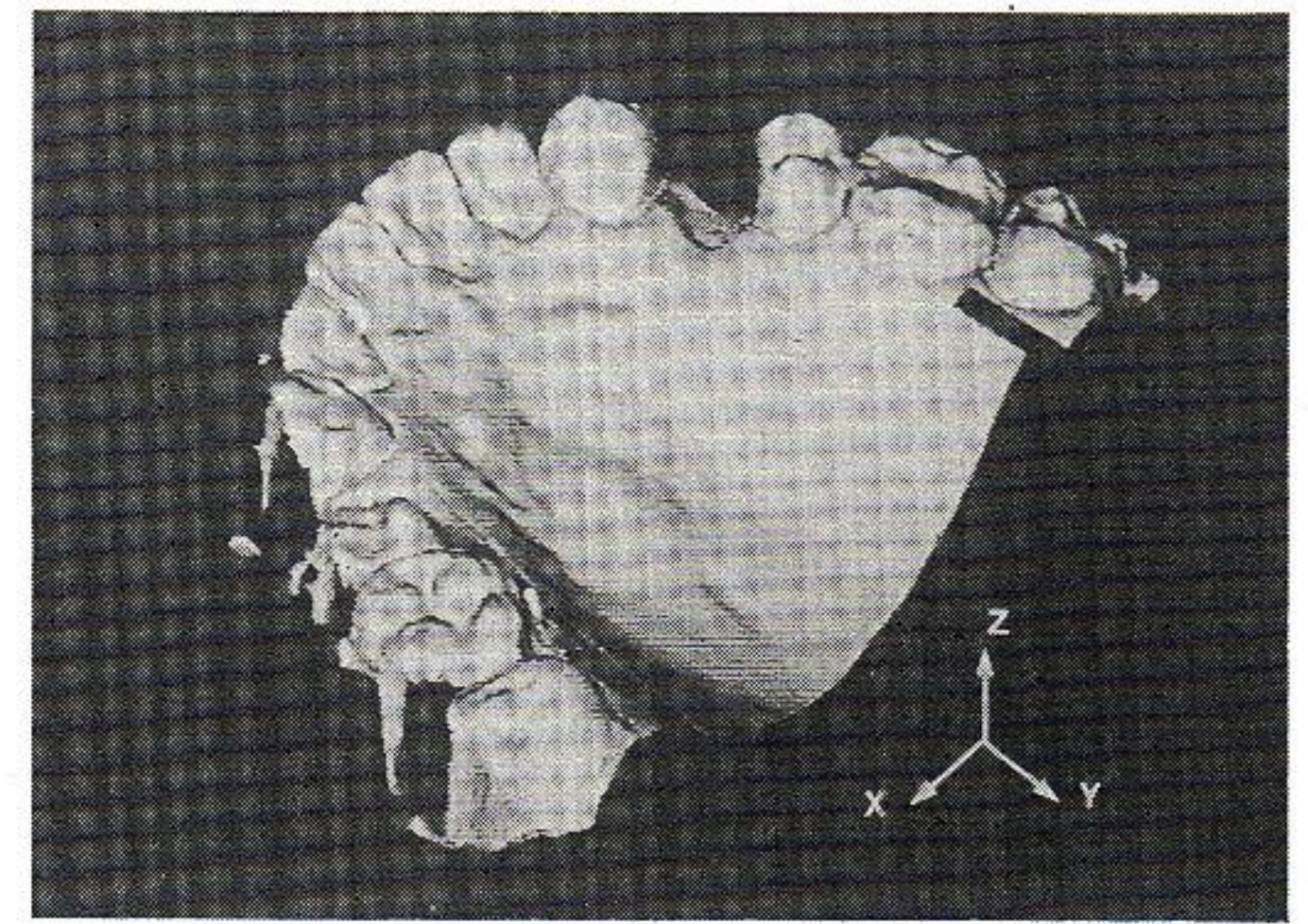

Fig. 3. Computer graphic display, using a shading method, of cast profiles of the patient's maxilla measured by the present system. Teeth and the palate were measured in a pitch of 0.2 and $0.4 \mathrm{~mm}$, respectively. The first premolars of this patient were extracted prior to orthodontic treatment.

$\pm 12 \mathrm{~mm}$, a spot on a plaster block fixed on a mechanical $Z$-stage. Since the relation is slightly curvilinear in principle, the regression curve of the 50 measured data was obtained and used for converting the peak position into depth. The standard deviation of the data around the curve was $0.024 \mathrm{~mm}$, and $92 \%$ of the data distributed with an error of less than $0.05 \mathrm{~mm}$. The present apparatus is, therefore, able to detect a depth with an approximate accuracy of $0.05 \mathrm{~mm}$.

We also tested the lateral resolution by scanning a plaster block on which thin grooves were cast using metal plates of known thickness $(0.1$ and $0.2 \mathrm{~mm})$ and confirmed that our system attained the lateral resolution of at least $0.1 \mathrm{~mm}$. In our current system, actual resolution is not determined by the sharpened laser beam but by the minimum scanning pitch $0.1 \mathrm{~mm}$ of the plotter used.

\section{Measurement of Three-Dimensional Tooth MOVEMENT}

\section{Numerical Registration of Three-Dimensional Image}

Our principle of measuring the three-dimensional movement of a tooth during orthodontic treatment is based on the registration, using a computer, of three-dimensional tooth images measured by the present profilometer at different stages of treatment. Namely, tooth profiles taken at the initial stage of treatment are numerically moved and superimposed on those of a certain later stage. The numerical movement of each tooth which attains to the close matching of the images gives the actual movement of the tooth.

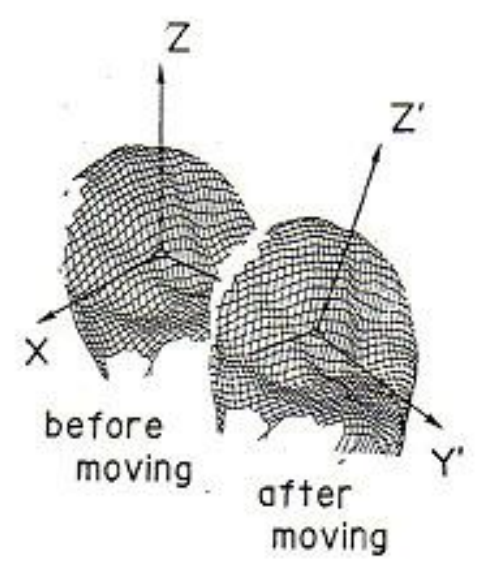

(a)

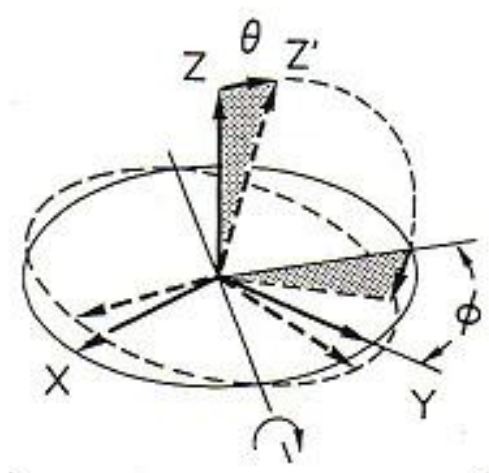

(c)

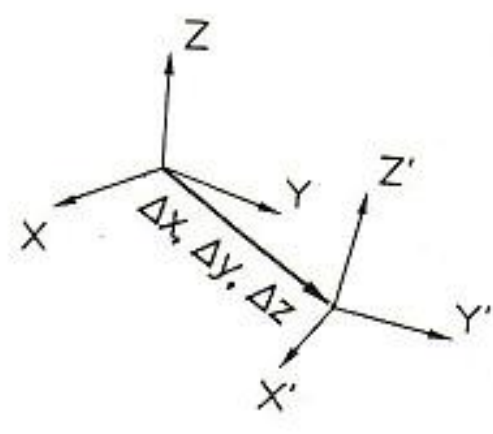

(b)

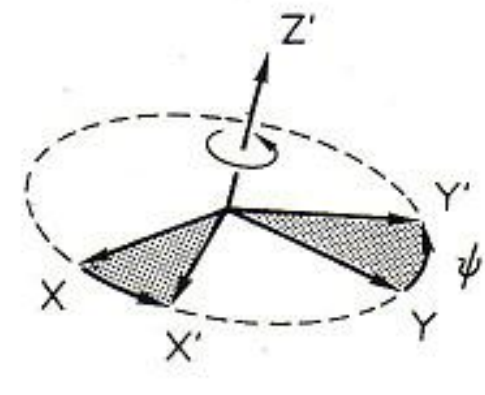

Fig. 4. Coordinate system for calculating tooth movement by registration. (a) The coordinate system fixed on a tooth. The origin was chosen as the centroid of the occlusal surface data. (b) Translations denoted by $\Delta x, \Delta y$, and $\Delta z$. (c) Tipping (left) and rotation (right). $\phi$ is direction of tipping, $\theta$ angle of tipping, and $\psi$ rotation with respect to $Z$-axis. Directions of axes are defined as in Fig. 3.

In order to represent changes in position of a rigid body in three-dimensional space, we must introduce six parameters. These are defined, as illustrated in Fig. 4, by the translations $\Delta x, \Delta y$ and $\Delta z$, and by the rotations $\phi, \theta$, and $\psi$ which denote the tipping direction, the tipping angle and the rotation angle with respect to the $Z$-axis, respectively. In conformity with the terms tipping and rotation defined in dentistry, the present definition of the angles were modified from Euler's angles conventionally used for describing the amount of rotation in three-dimensional space, i.e., $\psi$ was redefined by $\phi+\psi$ of Euler's angles. According to this definition, a tooth tips by $\theta$ to the direction of $\phi$ and then rotates by $\psi$ around the $Z^{\prime}$-axis, as shown in Fig. 4. The coordinate system was fixed on each tooth of the initial stage and its origin was chosen as the centroid of the occlusal surface determined from the tooth profiles of the initial stage.

New coordinates necessary for carrying out the registration were calculated by the following equation:

$$
\mathbb{u}^{\prime}=\mathbb{R}^{\prime}+\Delta \mathbb{r}^{\prime}
$$

where $u^{\prime}$ is the coordinates $\left[x^{\prime}, y^{\prime}, z^{\prime}\right]^{T}$ after the numerical moving, $\left[s\right.$ is a rotation matrix, $\nabla^{\prime}$ is the original $[x, y, z]^{T}$ before the moving, and $\Delta v^{P}$ is the translations $[\Delta x, \Delta y, \Delta z]^{T}$. The in iven by the matrix:

$$
\left[\begin{array}{ccc}
\cos \theta \cos \phi \cos (\psi-\phi) & -\cos \theta \cos \phi \sin (\psi-\phi) & \sin \theta \cos \phi \\
-\sin \phi \sin (\psi-\phi) & -\sin \phi \cos (\psi-\phi) & \\
\cos \theta \sin \phi \cos (\psi-\phi) & -\cos \theta \sin \phi \sin (\psi-\phi) & \sin \theta \sin \phi \\
+\cos \phi \sin (\psi-\phi) & +\cos \phi \cos (\psi-\phi) & \\
-\sin \theta \cos (\psi-\phi) & \sin \theta \sin (\psi-\phi) & \cos \theta
\end{array}\right]
$$


The registration was carried out automatically by searching for an optimal combination of 4 and $\Delta$ until the amount of mismatching of the tooth images of the initial and the later stages became minimum. The mismatching was evaluated from the averaged value of difference $u$ in the $z$-coordinate.

$$
u=\sum_{i=1}^{n}\left|z_{i}^{\prime}-z_{i}\right| / n
$$

where $z_{i}^{\prime}$ denotes the numerically moved datum $z^{\prime}\left(x^{\prime}, y^{\prime}\right)$, calculated by (1), of the initial stage, $z_{i}$ is the datum of the later stage, and $n$ is the number of data used for the registration. For the coincidence of horizontal coordinates of $z_{i}^{\prime}$ and $z_{i}, z_{i}$ was interpolated; $z\left(x^{\prime}, y^{\prime}\right)$ was obtained from four values of $z(x, y)$ around the point $\left(x^{\prime}, y^{\prime}\right)$. Mathematically the registration is regarded as a procedure searching for a point of minimum of a function of multivariable. Such a procedure was executed by an iterative method of calculation, changing the value of one of the six parameters back and forth, and repeating the same algorithm is turn on the parameters to reach the final minimum of the function $u$. At the beginning of the iteration we set initial values of the parameters to $1 \mathrm{~mm}$ in translations, $5^{\circ}$ in $\theta$ and $\psi$, and $15^{\circ}$ in $\phi$. These initial values were not so critical in finding the minimum. But, coarse alignment in translations using the centroid of the profiles before the iteration was effective in preventing the above procedure from reaching a false, local minimum.

Prior to the registration of teeth, the three-dimensional position of casts to be compared must be normalized, because the position cannot be adjusted accurately when casts are measured. For this purpose, the palate was employed as a reference. Namely, the palates were first superimposed by the same algorithm as that of teeth. As described later in "Discussion," profile details of the palate are rather stable during orthodontic treatment and therefore appropriate for an immovable reference.

The error in the calculated movement by the above procedure was evaluated through the simulation study, in which actual measured data of a molar were numerically moved by substituting random numbers for the parameters and then the registration was performed between the measured and the moved data. The 36 movements calculated in all showed sufficiently small errors less than $0.1 \mathrm{~mm}$ in translation and $0.5^{\circ}$ in rotation. It should be noted that the error in translation is less than the scanning pitch $0.2 \mathrm{~mm}$ of a tooth because the procedure in the registration was designed to interpolate the data.

\section{Clinical Measurement}

Various types of springs are in common use as an orthodontic appliance to produce a force on teeth. In order to quantify the effect of spring configurations on tooth movement, we measured upper canine movements on patients over periods of 41 to 190 days when the canines were moved by two types of springs conventionally used: an open single loop (referred to as spring $A$ ) and a closed loop with multiple helices (spring $B$ ), which are illustrated in Fig. 5. After extracting the first premolars, canine retraction was commenced on three males and seven females. Patients were $11-15$ years of age, with the exception of a 23-year-old male. A force of $150 \mathrm{~g}$ was applied so that the posterior teeth pulled the canine by the sectional retraction method. As the force decreases with space closure, the springs were reactivated to $150 \mathrm{~g}$ almost every month. At that time a dental cast was taken from a patient and the canine move-

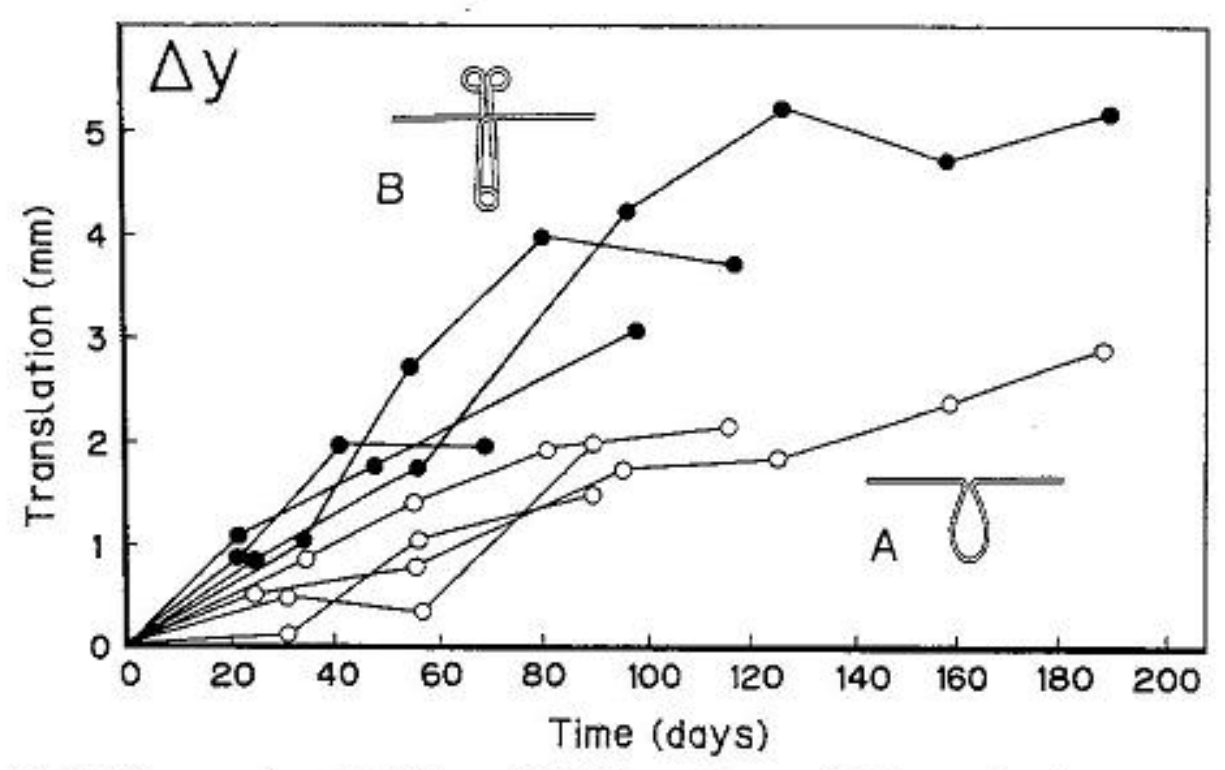

Fig. 5. Time course analysis of tooth movement during orthodontic treatment. Canine translations $\Delta y$ to a posterior side are plotted. Open and closed circles show the movement by using springs $A$ and $B$, respectively.

ment from the initial stage to that stage was measured by the procedure mentioned in the previous section.

Data were collected under four groups according to the treatment conditions classified below. In connection with this, two kinds of bends incorporated in a spring should be referred to. When a single force is placed on a lateral portion of the crown of a tooth, the tooth rotates and tips owing to the moment of rotation resulting from the force. Against such a rotational tendency antirotation and gable bends are commonly set up in a spring to create a moment of counter-rotation. The magnitude of this effective force is adjusted by an angle of bending. Classified treatment conditions are the following. In all groups the angle of a gable bend was set to $20-35^{\circ}$

Group 1: spring $A$ with an antirotation angle of $20^{\circ}$.

Group 2: spring $B$ with the same bend as the above.

Group 3: spring $A$ without an antirotation bend.

Group 4: spring $B$ without an antirotation bend.

As shown in Fig. 5, the spring $B$ (group 2) is capable of moving the canine almost twice as fast as the spring $A$ (group 1) because spring $B$, which has the multiple helices and numerous flexibility, conserves most of the activated force even if a certain amount of sace closure occurs. However, spring $B$ (groups 2 and 4) shows no significant effect of the antirotation bend on tooth rotation $\psi$, as shown in Fig. 6. On the contrary, spring $A$, which has less flexibility than spring $B$, has the greater ability to protect the rotation when incorporating the antirotation bend (group 1), as shown in Fig. 7.

\section{Discussion}

An apparatus using an optical probe, developed by Berkowitz et al. [8], is the first and the only automatic apparatus, to our knowledge, for three-dimensional measurement of dental casts. Although it was successfully applied to cleft palate cast analysis, it has the limitation of the minimum detectable depth interval of $0.5 \mathrm{~mm}$ and the lateral resolution of about $1 \mathrm{~mm}$ under the total measuring depth range of $18 \mathrm{~mm}$. It is because the measurement is based on utilizing the relationship between the depth and the light intensity change due to defocusing. The recent development of the travelling microscope by Bhatia and Harrison [7] has notably increased the accuracy of measurement in $Z$-dimension. The claimed accuracy is $0.024 \mathrm{~mm}$, but this apparatus has to be operated manually as well as the others [4][6]. The present apparatus is automated and its accuracy is, at least, $0.05 \mathrm{~mm}$ in depth and $0.1 \mathrm{~mm}$ on a horizontal plane within 


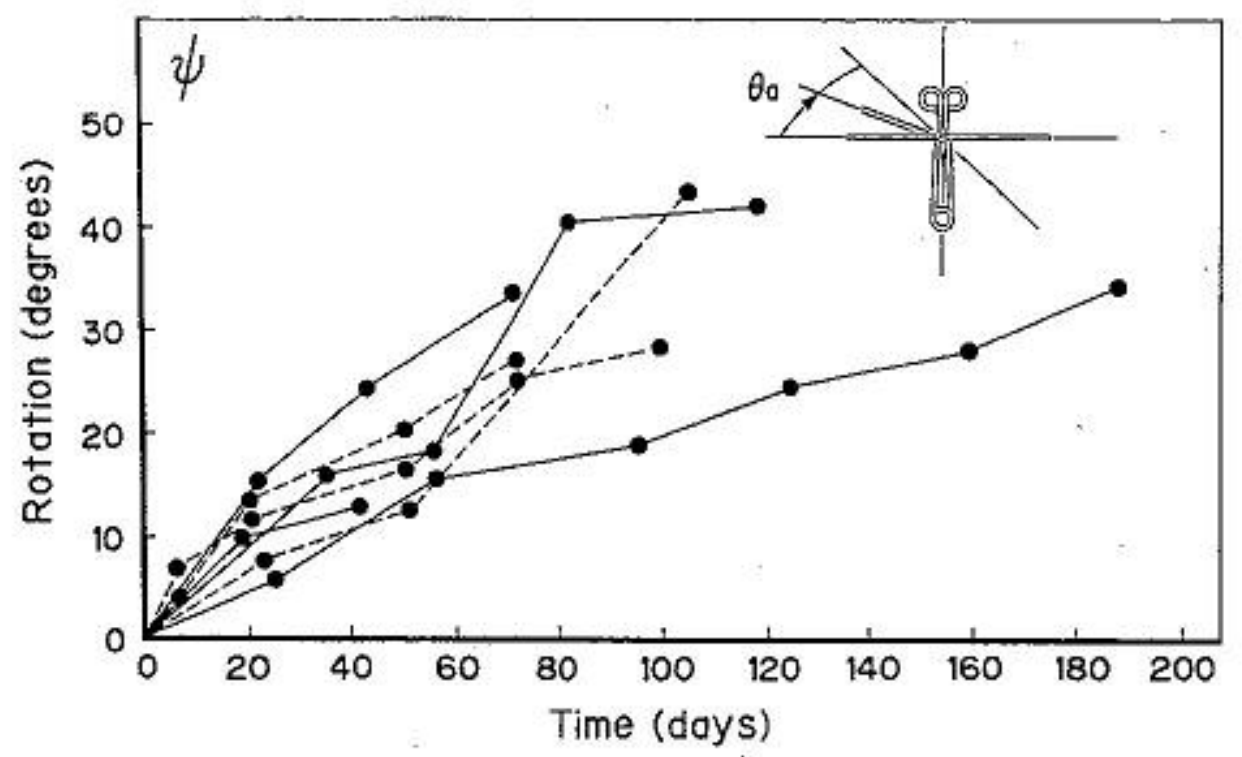

Fig. 6. Rotation of the canines $\psi$ around $Z$-axis during orthodontic treatment by spring $B$. No significant difference was observed between the groups with the antirotation bend $\theta_{a}$ of $20^{\circ}$ (solid lines) and without a bend (broken lines).

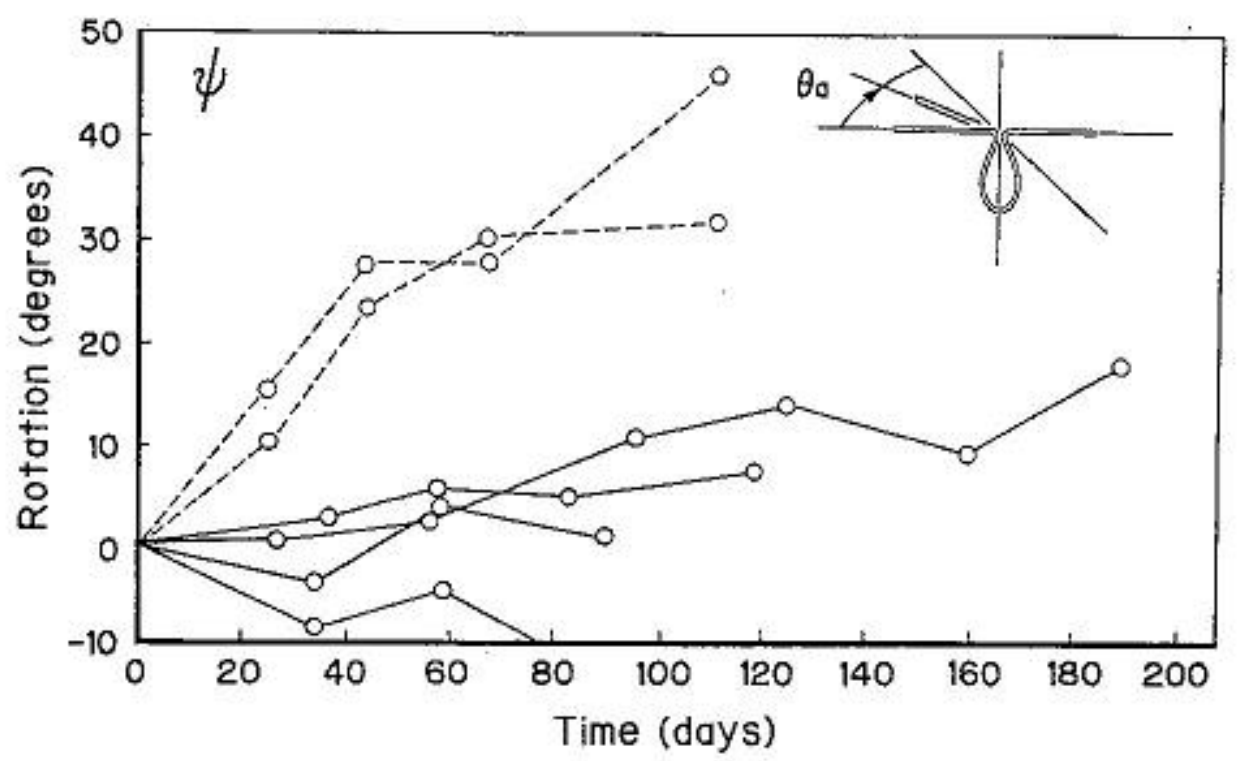

Fig. 7. Effect of an antirotation bend in spring $A$ on the tooth rotation $\psi$. The spring with the antirotation bend $\theta_{a}$ of $20^{\circ}$ (solid lines) showed the marked effect in comparison with the spring without a bend (broken lines). Negative values mean the counter-rotation caused by the antirotation bend.

the depth range of $25 \mathrm{~mm}$. The problem to be overcome is that data of the lateral portion of a tooth are unavailable because the optical equipment is placed on the side above a dental cast, as is most apparatus developed up to now.

Previous studies with respect to the measurement of tooth movement over a long period of orthodontic treatment were restricted to one or two dimensions. As for the three-dimensional measurement, only the instantaneous translation and rotation, observed immediately after applying an orthodontic force to a tooth, have been measured using laser hologram interferometry [13]-[16]. This technique measures movement with the highest degree of sensitivity but is restricted to short-term observations. Our approach is based on registration of profiles of serial casts, and consequently enables long-term movement to be followed. An interesting method also using registration for the measurement of two-dimensional tooth movement during orthodontic treatment has recently been reported [17] where a cast and a holographic image representing different stages are manually superimposed within an $X-Y$ plane by mechanical stages.

The accuracy of movement measurement with the present method depends on the registration and the immovable reference. The error in the registration examined by the simulation study was less than $0.1 \mathrm{~mm}$ in translation and $0.5^{\circ}$ in rotation. These are, however, the minimum errors resulting only from the calculation. Actual accuracy is mostly determined by the reproducibility of plaster casts. Therefore, care should be taken in the process of making a cast. The effect of the reproducibility on the error is now being studied. To confirm the morphological stability of the palate employed as a reference, we evaluated its deformation during orthodontic treatments of 6-21 months in nine patients of 8-14 years of age. The "averaged" value of difference in depth on each patient before and after treatment was only $0.05-0.13 \mathrm{~mm}$, which were calculated by (2) except for the palate data around realigned teeth. The deformation of the palate around the teeth is much larger than that of the bottom. In practice we have used palate data within 5 $\mathrm{mm}$ from the bottom as the reference. The palate is, thus, an appropriate reference. However, it has limitation of applicability only to the maxilla because of the presence of the tongue in the mandible.

Despite the above limitation, the results in the time course analysis of tooth movement on the patients suggest the usefulness of the present technique in quantifying the action of a spring upon tooth movement. The importance of such a technique is easily understood from the fact that it is quite difficult to detect three-dimensional tooth movement with the eyes. The present technique also shows promise of presenting useful quantitative information to the orthodontist, who is then able to optimize a treatment procedure by readjustment of the magnitude and direction of the force and of the antirotation and gable bends of a spring in response to the tooth movement due to treatment. The automatic digitization of cast profiles will permit further application to computerization of cast analysis [18], which is now performed on manually digitized data.

\section{ACKNOWLEDGMENT}

The authors are grateful to Dr. R. Harada and H. Ohnuma for their assistance in the construction of the prototype and to A. Toshimitsu for his assistance in the numerical calculation.

\section{REFERENCES}

[1] B. S. Savara, "Applications of photogrammetry for quantitative study of tooth and face morphology," Amer. J. Phys. Anthropol., vol. 23, pp. 427-434, 1965.

[2] S. Berkowitz and S. Pruzansky, "Stereophotogrammetry of serial casts of cleft palate," Angle Orthod., vol. 38, pp. 136-149, 1968.

[3] S. Berkowitz, "Stereophotogrammetric analysis of casts of normal and abnormal palates," Amer, J. Orthod., vol. 60, pp.1-18, 1971.

[4] F. P. G. M. van der Linden, H. Boersma, T. Zelders, K. A. Peters, and J. H. Raaben, "Three-dimensional analysis of dental casts by means of the Optocom," J. Dent. Res., vol. 51, p. 1100 , 1972.

[5] G. W. Butcher and C. D. Stephens, "The reflex optical plotterA preliminary report," Brit. Dent. J., vol. 151, pp. 304-305, 1981.

[6] K. Takada, A. A. Lowe, and R. DeCou, "Operational performance of the Reflex Metrograph and its applicability to the threedimensional analysis of dental casts," Amer. J. Orthod., vol. 83, pp. 195-199, 1983.

[7] S. N. Bhatia and V. E. Harrison, "Operational performance of the travelling microscope in the measurement of dental casts," Brit. J. Orthod., vol. 14, pp. 147-153, 1987.

[8] S. Berkowitz, G. Gonzalez, and L. Nghiem-phu, "An optical profilometer-A new instrument for the three dimensional measurement of cleft palate casts," Cleft Palate J., vol. 19, pp. 129$138, ' 1982$

[9] R. Harada, K. Yamamoto, H. Ohnuma, T. Mikami, and S. Nakamura, "Three-dimensional measurement of dental cast using laser and image-sensor (in Japanese)," Jap. J. Med. Elect. Biol. Eng., vol. 23, pp. 166-171, 1985.

[10] K. Yamamoto, A. Toshimitsu, T. Mikami, S. Hayashi, R. Harada, and S. Nakamura, "Optical Measurement of Dental cast profile and application to analysis of three-dimensional tooth 
movement in orthodontics," Frontiers Med. Biol. Eng., vol. 1, pp. 119-130, 1988.

[11] R. M. Ricketts, R. W. Bench, J. J. Hilgers, and R. Schulhof, "An overview of computerized cephalometrics," Amer. J. Orthod., vol. 61, pp. 1-28, 1972.

[12] A. Ishida, S. Suzuki, S. Imai, and Y. Mori, "Scoliosis evaluation utilizing truncal cross-sections," Med. Biol. Eng. Comput., vol. 20 , pp. 181-186, 1982.

[13] H. Ryden, H. Bjelkhagen, and P. Soder, "'The use of laser beams for measuring tooth mobility and tooth movement-An in vitro study," J. Periodontol., vol. 45, pp. 283-287, 1974.

[14] P. R. Wedendal and H. I. Bjelkhagen, "Dynamics of human teeth in function by means of double pulsed holography-An experimental investigation," Appl. Opt., vol. 13, pp. 2481-2485, 1974.

[15] R. J. Pryputniewicz, C. J. Burstone, and W. W. Bowley, "Determination of arbitrary tooth displacements, ' J. Dent. Res., vol. 57, pp. 663-674, 1978.

[16] C. J. Burstone, R. J. Pryputniewicz, and W. W. Bowley, “Holographic measurement of tooth mobility in three dimensions," J. Periodontal Res., vol. 13, pp. 283-294, 1978.

[17] H. Ryden, H. Bjelkhagen, and B. Martensson, "Tooth position measurements on dental casts using holographic images," Amer. J. Orthod., vol. 81, pp. 310-313, 1982.

[18] E. A. BeGole, J. F. Cleall, and H. C. Gorny, "A computer system for the analysis of dental casts," Angle Orthod., vol. 51, pp. 252-258, 1981.

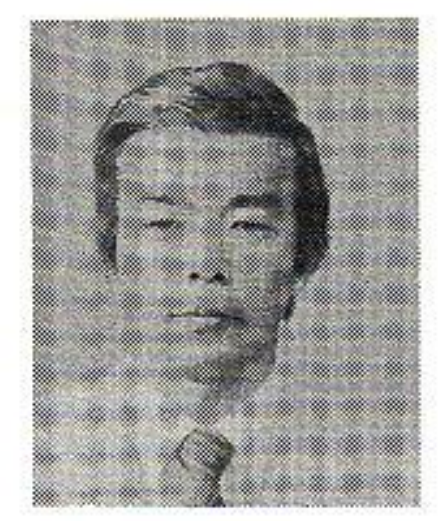

Katsuyuki Yamamoto (M'90) received the B.S., M.S., and Ph.D. degrees in electronic engineering from Hokkaido University, Sapporo, Japan, in 1969,1971 , and 1975 , respectively.

From 1975 to 1987 he was an Instructor at the Research Institute of Applied Electricity, Hokkaido University. Since 1987 he has been an Associate Professor of Biomedical Engineering at the Faculty of Engineering, Hokkaido University. His current research interests are in noninvasive measurements using ultrasonic and optical techniques, biomedical signal processing, and medical engineering in dentistry.

Dr. Yamamoto is a member of the Japan Society of Medical Electronics and Biological Engineering, the Institute of Electronics, Information, and Communication Engineers, the Japan Society of Ultrasonics in Medicine, and the International Society of Artificial Organs.

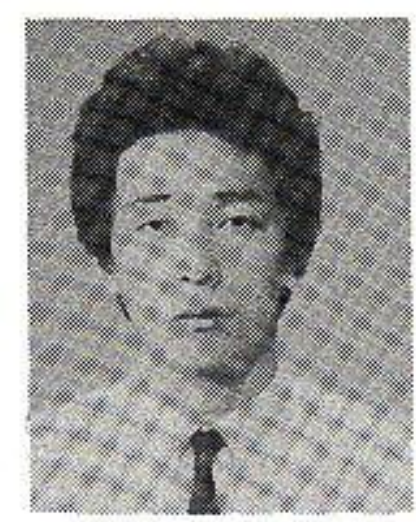

Syunsuke Hayashi received the D.D.S. and $\mathrm{Ph} . \mathrm{D}$. degrees in dental science from Hokkaido University, Sapporo, Japan, in 1984 and 1988, respectively.

He was a member on the staff of the Hokkaido University Dental Hospital and was a Research Associate at Higashi-Nippon-Gakuen University School of Dentistry. Since 1989 he has been a dental practitioner at his own hospital. His research interest is in movement analysis of the teeth during orthodontic treat-
Dr. Hayashi is a member of the Japan Orthodontic Society and the Japan Society of Medical Electronics and Biological Engineering.

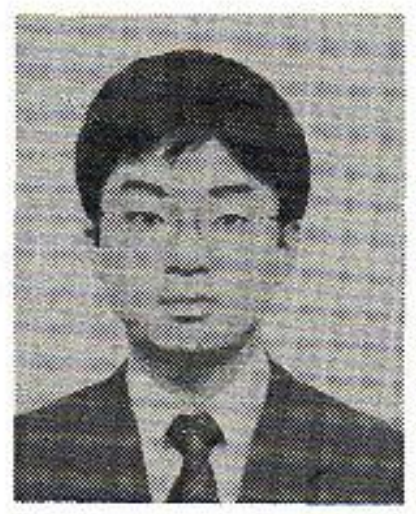

Hirofumi Nishikawa received the B.S. degree in information engineering from Niigata University, Niigata, Japan, in 1987 and the M.S. degree in biomedical engineering from Hokkaido University, Sapporo, Japan in 1989.

Since 1989 he has been employed as a research engineer at Communication System Development Laboratory, Mitsubishi Electric Corporation, where he is working on high speed image processing.

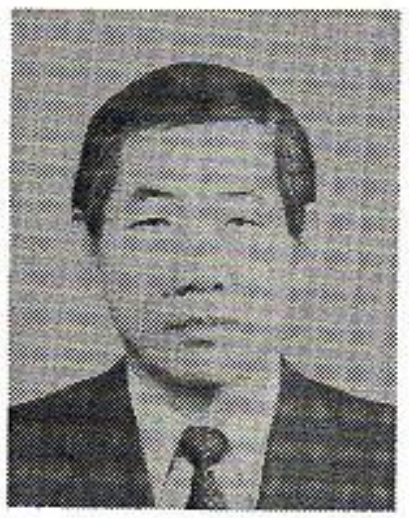

Shinji Nakamura received the D.D.S. and Ph.D. degrees in dental science from Tokyo Medical and Dental University, Tokyo, Japan, in 1964 and 1968 , respectively.

From 1968 to 1970 he was an Instructor at the School of Dentistry, Oregon Health Science University. In 1970, he returned to Tokyo Medical and Dental University as an Research Associate. In 1971, he joined the School of Dentistry at Hokkaido University as an Assistant Professor, where he has been Professor and Chairman of the Department of Orthodontics since 1975. His current activities in research include computerized orthodontic diagnosis and orthopedic approach to patients with skeletal malocclusion.

Dr. Nakamura is a member of the Japan Orthodontic Society, the American Association of Orthodontics, the Japanese Cleft Palate Association, and the Japan Society of Medical Electronics and Biological Engineering.

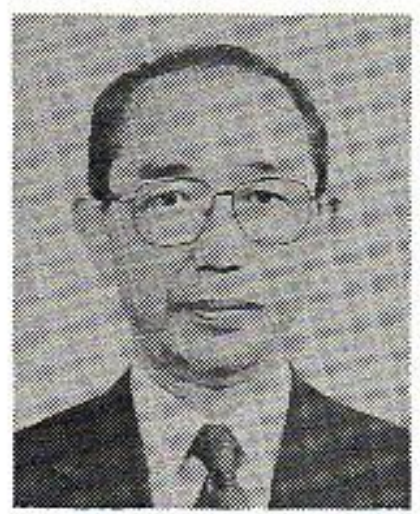

Tomohisa Mikami received the B.S. degree in physics and the Ph.D. degree in medical science from Hokkaido University, Sapporo, Japan, in 1951 and 1959, respectively.

From 1962 to 1986 he worked at the Research Institute of Applied Electricity, Hokkaido University, as an Associate Professor, and as a Professor from 1971. In 1986 he joined the Faculty of Engineering, Hokkaido University, where he is currently a-Professor of Biomedical Engineering. His current activities in research include systems analysis of respiration and circulation, optical and ultrasonic measurements for the body, and biomedical information processing.

Dr. Mikami is a member of the International Society for Artificial Organs, the Japan Society of Medical Electronics and Biological Engineering, the Japanese Society for Artificial Organs, and the Institute of Electronics, Information, and Communication Engineers. 